\title{
Immune complexes exposed on mast cell-derived nanovesicles amplify allergic inflammation
}

To the Editor,

Extracellular vesicles (EVs) are released by different cell types including mast cells (MCs) and are involved in intercellular communication thanks to their complex cargo of bioactive molecules.

Among them, exosomes represent nanovesicles (<150 nm) secreted from endosomal compartments upon their fusion with the plasma membrane. $^{1}$

Through both constitutive and induced exocytosis, MCs release exosomes that deliver several molecules including mRNA/miRNA ${ }^{2}$ and subunits of the high-affinity IgE receptor (Fc\&RI). ${ }^{3}$ However, the role of $\mathrm{MC}$-derived vesicles in the context of allergic reactions is still controversial. A recent study provides evidence that exosomes released from unstimulated mouse bone marrow-derived mast cells (mBMMCs) bind to free IgE and decrease circulating IgE levels, thus inhibiting the allergic response. ${ }^{4}$ In contrast, several evidences support a positive regulatory role of $\mathrm{MC}$-derived exosomes, ${ }^{5-7}$ suggesting that depending on MC activation status they can either attenuate or stimulate the inflammatory responses.

To gain insight into this issue, we isolated nanovesicles from $\mathrm{mB}$ MMCs (Figure 1) and rat basophilic leukemia cell line RBL-2H3 culture supernatants (Figure S1) by sequential ultracentrifugation, and characterized them by electron microscopy, flow cytometer, and dynamic light scattering (DLS). Vesicles displayed a cup-shaped morphology and a size of $100 \mathrm{~nm}$ (Figure 1A and Figure S1A, left panels), resulted in the enrichment of the exosomal markers CD63 and CD81 while they did not express calreticulin (Figure 1A and Figure S1A, right panels, and Figure S1B, C), and showed a Gaussian size distribution centered around $100 \mathrm{~nm}$ (Figure 1B and Figure S1D). These data demonstrate that our purification protocol provides an effective method for the enrichment of exosome-like vesicles.

To demonstrate the endosomal origin of purified nanovesicles, we silenced the expression of $\mathrm{Hrs}$, a member of the endosomal sorting complex required for transport (ESCRT), ${ }^{8}$ and quantified exosomes by DLS, as detailed in Figure S2A-C. Comparing to control cells, we observed a dramatic decrease in the number of vesicles released by Hrs-depleted cells (Figure S2D, E), demonstrating the indispensable role of Hrs in MC-derived exosome biogenesis.

To analyze the impact of $\mathrm{F} c \varepsilon \mathrm{RI}$ crosslinking on nanovesicle release, mBMMCs were loaded with anti-DNP IgE and stimulated with the specific multivalent Ag DNP-HSA. Quantification of exosome-like vesicles purified from untreated and treated mBMMCs (hereafter referred as EXO-NT and EXO-T, respectively) demonstrated that Ag-mediated receptor engagement greatly increases vesicle secretion, without a significant change in size distribution (Figure 1C). Of note, only EXO-T displayed IgE and Ag on their surface, as revealed by FACS analysis (Figure 1D).

Similar results were obtained on RBL-2H3 cells (Figure S3A, B). Moreover, exosome-like vesicles purified from both untreated and treated RBL-2H3 cells contained FceRI subunits, as previously reported, ${ }^{3}$ but we also found that $\mathrm{Ag}$ stimulation significantly increased the basal amount of $\beta$ chains (Figure S3C). Since we have previously demonstrated that engaged Fc\&RI receptors are internalized and routed to lysosomes, ${ }^{9}$ our results support the conclusion that a pool of internalized receptor complexes undergo a fate different from degradation being released into exosome-like vesicles together with IgE and Ags.

To evaluate the contribution of those complexes in vesicle transfer to other MCs, equal amounts of EXO-NT and EXO-T were incubated with IgE-loaded RBL-2H3 cells after fluorescent staining. As revealed by FACS, only EXO-T were efficiently taken up by IgE-loaded MCs and the uptake was inhibited in the presence of the high-affinity monovalent Ag DNP-Lysine, capable of breaking up aggregates induced by multivalent $\mathrm{Ag}$ (Figure S4A, B). Confocal microscopy experiments revealed the presence of internalized red-labeled vesicles (Figure S4C). Furthermore, pretreatment of MCs with dynasore, a specific inhibitor of clathrin-dependent internalization, strongly reduced vesicle uptake (Figure S4D), demonstrating that endocytosis is involved in this process. Thus, we conclude that the uptake of exosome-like vesicles by sensitized MCs mainly occurs by endocytosis through the binding between $\mathrm{Ag}$ displayed on vesicles and IgE bound on MCs.

To investigate the functional role of Ag-bearing vesicles, EXO-NT and EXO-T were incubated with IgE-loaded MCs. Only EXO-T promoted the release of $\beta$-hexosaminidase, a marker of $M C$ degranulation (Figure 1E), and induced cytokine production (Figure $1 \mathrm{~F}$ ) at level comparable to that obtained by using soluble multivalent $\mathrm{Ag}$ (Figure S5). Stimulation in the presence of DNP-Lysine dramatically reduced degranulation and cytokine production (Figure 1E, F and Figure S5).

Similar results were obtained by using RBL-2H3-derived exosomes (data not shown).

Abbreviations: Ag, antigen; DC, dendritic ell; DcLS, dynamic light scattering; DNP-HSA, dinitrophenyl-human serum albumin; ESCRT, endosomal sorting complex required for transport; EV, extracellular vesicle; EXO-NT, EXOsome released from uNTreated cells; EXO-T, EXOsomes released from Treated cells; FcERI, high-affinity Fc Receptor for IgE; Hrs, hepatocyte growth factor-regulated tyrosine kinase substrate; IL, interleukin; mBMMC, mouse bone marrow-derived mast cells; MC, mast cells; MVB, multivesicular bodies; TEM, transmission electron microscopy. 
(A)
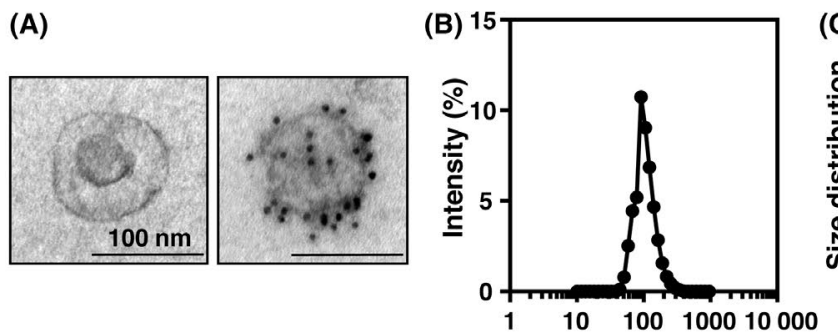

Hydrodynamic diameter $(\mathrm{nm})$

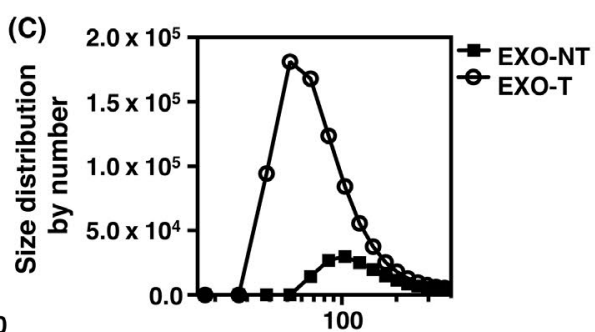

Hydrodynamic diameter $(\mathrm{nm})$

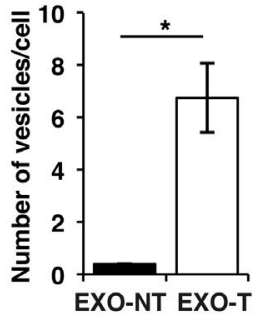

EXO-NT EXO-T
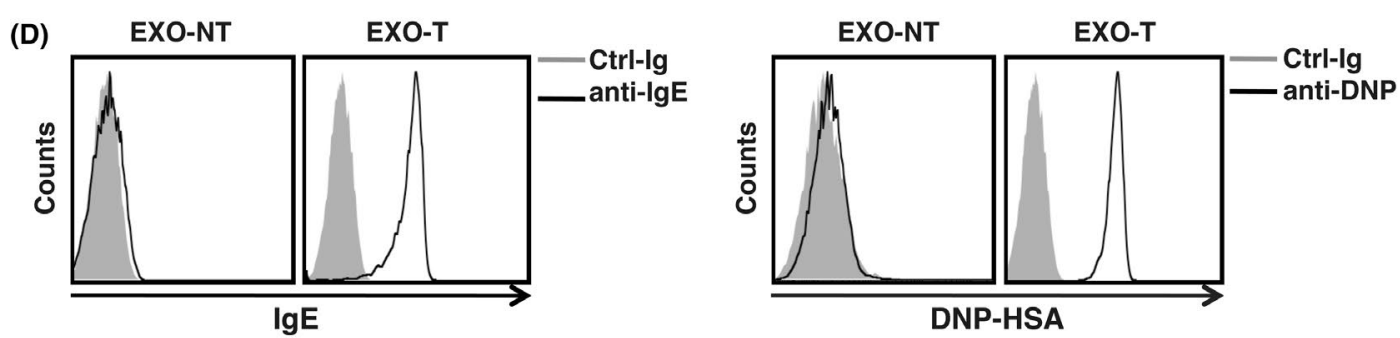

(E)

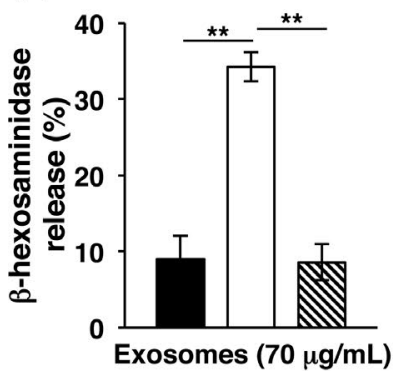

(F)

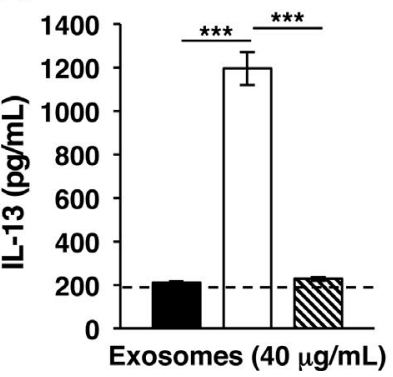

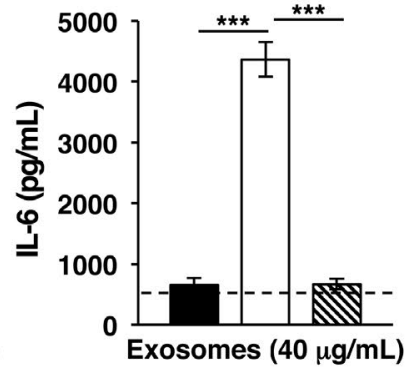

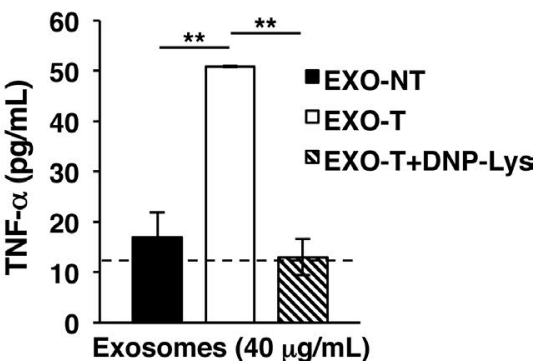

FIGURE 1 Characterization of nanovesicles released by mouse mast cells. Nanovesicles were purified from culture supernatants of untreated (EXO-NT) and IgE and antigen-treated (EXO-T) mBMMCs. A, Transmission electron microscopy (TEM) (left panel) and immunogold labeling using anti-CD63 Ab (right panel). Results are representative of three independent experiments. B and C, Nanovesicle size analysis (B) and quantification (C, left panel) by dynamic light scattering (DLS). The number of vesicles per cell is shown as mean \pm SD of three independent measures (C, right panel). ${ }^{*} P<.05$, Student's $t$ test. D, Equal amount $(10 \mu \mathrm{g})$ of EXO-NT or EXO-T was passively adsorbed on $4 \mu \mathrm{m}$ aldehyde/sulfate latex beads. IgE and Ag expression was evaluated by FACS using FITC-conjugated anti-IgE or Alexa 488-conjugated anti-DNP (empty histograms) Abs. Filled histograms represent staining with isotype control Abs (Ctrl lg). Results are representative of three independent experiments. E and F, Mouse BMMCs were loaded with anti-DNP IgE mAb and incubated with exosome-like vesicles (EXONT or EXO-T). Upon $1 \mathrm{~min}$ of incubation $50 \mu \mathrm{mol} / \mathrm{L}$ DNP-Lys was added, where indicated. After $30 \mathrm{~min}, \beta$-hexosaminidase release was measured in culture supernatants (E). After $6 \mathrm{~h}$, supernatants were collected and cytokines were measured by multiplex cytokine assay (F). Dashed lines indicate the cytokine amounts released by unstimulated cells. All data are presented as the mean \pm SD from three independent experiments. ${ }^{* *} P<.01,{ }^{* * *} P<.001$ one-way ANOVA with Tukey's multiple comparison test. EXO-NT, EXOsome released from uNTreated cells; EXO-T, EXOsomes released from Treated cells

Based on our findings, we propose that vesicle-associated Ags interact with FceRI-bound IgE promoting signaling and favoring vesicle uptake by endocytosis, thus representing a self-amplification mechanism that contributes to exacerbate local allergic reactions.

Fc\&RI-mediated vesicle uptake may also represent a mean to deliver Ags to other immune cells such as Fc\&RI-positive DCs, further contributing to allergic responses.

Finally, we investigated the presence of the $\mathrm{Fc \varepsilon RI- \alpha}$ subunit and IgE on nanovesicles purified from sera of atopic individuals with different total IgE levels (see Table S1). DLS analysis of vesicle preparation revealed a single peak of about $500 \mathrm{~nm}$ compatible with aggregates of 5-6 exosome-like vesicles with a diameter of 80$100 \mathrm{~nm}$ each (Figure 2A, B), while the presence of contaminating proteins was excluded (Figure 2B).
Undetectable IgE levels were found on vesicles purified from sera of nonatopic donors, while sera of atopic individuals displayed variable amounts of vesicle-associated IgE (Figure $2 \mathrm{C}$ ) that positively correlates with the serum IgE levels (Figure 2D).

Vesicles were also isolated from sera using anti-CD63 coated magnetic beads and analyzed by FACS (Figure 2E). Only nanovesicles from atopic individuals carried the $\mathrm{Fc \varepsilon RI}-\alpha$ chain (Figure $2 \mathrm{~F}$ ), supporting the conclusion that those nanovesicles display Fc\&RI/IgE complexes.

Due to the heterogeneity of sensitizing Ags among our donors' cohort, we were unable to verify whether those vesicles also deliver Ags. Studies are ongoing to compare nanovesicles derived from sera of atopic individuals before and during an acute phase of allergic reaction.

Collectively, our data uncovered a new role for nanovesicles derived from activated MCs in the positive regulation of allergic responses, as depicted in our working model (Figure S6). 
(A)

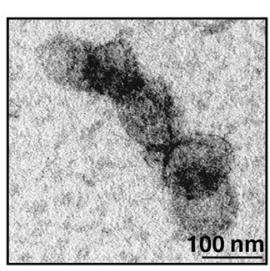

(C)

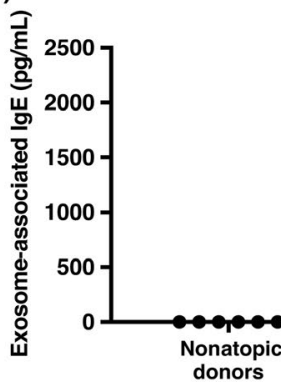

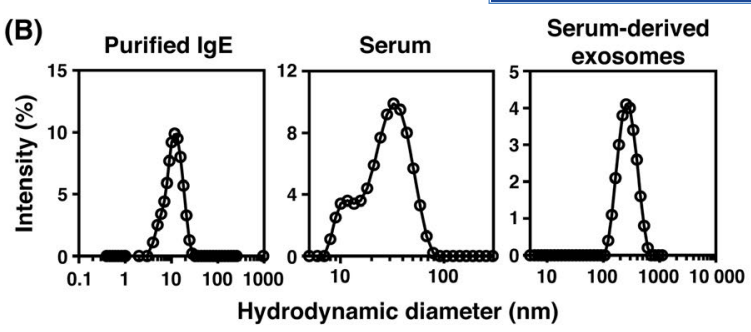

(D)

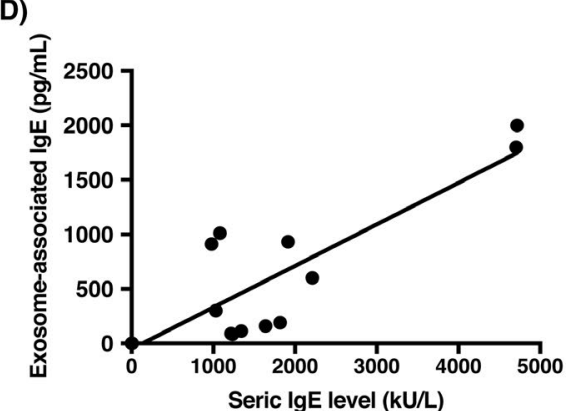

(E)

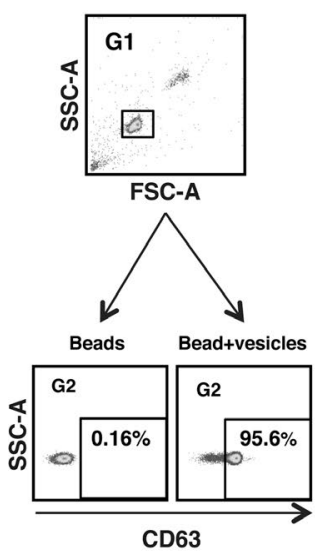

(F) Nonatopic donor \#5 Nonatopic donor \#6

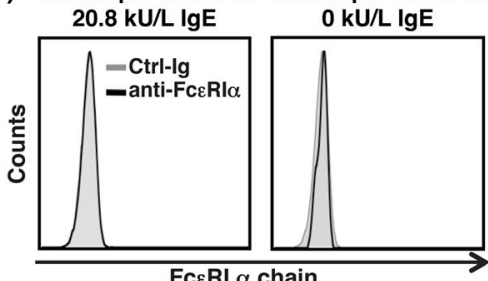

FceRl $\alpha$ chain

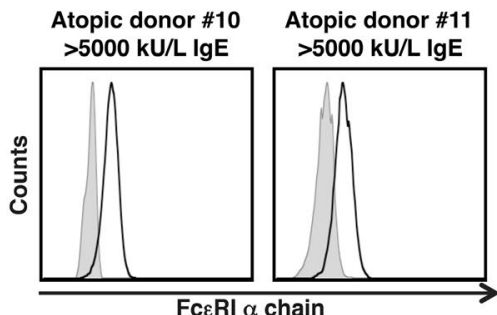

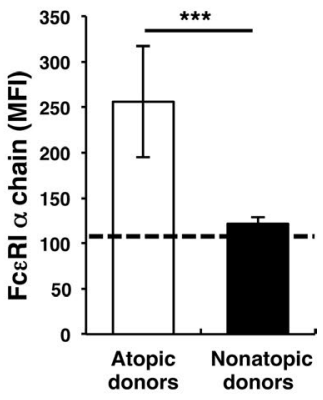

FIGURE 2 Characterization of exosome-like vesicles purified from sera of atopic donors. A, Representative TEM image of one aggregate composed of 5 vesicles isolated from human sera by ultracentrifugation. B, DLS analysis of purified IgE appearing as a single peak of about $10 \mathrm{~nm}$, human sera resulting in a series of peaks ranging from 10 to $80 \mathrm{~nm}$ likely representing proteins and protein aggregates, and serumderived vesicles appearing as a single peak of about $500 \mathrm{~nm}$. The results obtained for one representative donor is shown. $\mathrm{C}$, Nanovesicles were purified from sera of atopic $(n=12)$ or nonatopic donors $(n=6)$, and IgE measured by ELISA. Each dot represents a single donor. The means \pm SD are also shown. D, Correlation between IgE total level in patient's sera and IgE exposed by vesicles $\left(R^{2}=0.74 ; P<.0001\right)$. Each dot represents a single donor $(n=18)$. E, Nanovesicles were isolated from human sera by a first step of filtration and ultracentrifugation followed by incubation with CD63-coated magnetic beads. Bead-bound vesicles were stained with PE-conjugated anti-CD63. Beads were first gated on a forward scatter/side scatter (FSC-A/ SSC-A) dot plot to exclude debris and doublets (G1). Events in G1 were then visualized on SSC-A/CD63 dot plot, and CD63 ${ }^{+}$events were gated using beads without vesicles as negative control (G2). F, CD63 ${ }^{+}$vesicles were stained with BV510-conjugated anti-Fc\&RI- $\alpha$ chain mAb (empty histograms) or BV510-conjugated isotype Ctrl-lg (filled histograms) and then analyzed by FACS. Four representative donors are shown. Mean fluorescence intensity (MFI) of FceRI- $\alpha$ chain on exosomes is shown as mean \pm SD of atopic $(n=6)$ and nonatopic $(n=5)$ donors. Dashed lines represent the MFI of beads alone. ${ }^{* * *} P<.001$, Student's $t$ test

Understanding how these nanovesicles influence the hypersensitivity reactions could help the development of more efficient therapies.

\section{ACKNOWLEDGMENTS}

This work was supported by the Italian Ministry of Education, Universities and Research-Dipartimenti di Eccellenza - L. 232/2016 and the Pasteur Institute-Cenci Bolognetti Foundation-Call 2012. EV is supported by a fellowship from AIRC (Associazione Italiana per la Ricerca sul Cancro). We are grateful to Dr J.-P. Kinet for generous access to anti-Fc\&RI $\beta$ chain antibody and to $\mathrm{Dr}$ B. Wilson for helpful advices on exosome isolation. We thank $\mathrm{Dr}$ B. Zitti for isolating mouse bone marrow cells (mBMMCs), and Drs. G. Bernardini, F. Antonangeli, G. Sciumè and E. Sanseviero for technical advises on $\mathrm{mBMMC}$ culture. We also thank B. Milana for expert technical assistance, L. Simonelli for technical assistance in electron microscopy procedures and L. Cutini for IgE determination. The confocal fluorescence images were collected at the CLNS Imaging Facility of the Istituto Italiano di Tecnologia (Rome, Italy). 


\section{CONFLICT OF INTEREST}

The authors declare that they have no conflicts of interest.

$$
\begin{array}{r}
\text { Rosa Molfetta }^{1} \\
\text { Mario Lecce }^{1} \\
\text { Linda Quatrini }^{1} \\
\text { Giulio Caracciolo }^{1} \\
\text { Luca Digiacomo } \\
\text { Laura Masuelli }^{2} \\
\text { Nadia Domenica Milito } \\
\text { Elisabetta Vulpis }^{1} \\
\text { Alessandra } \text { Zingoni }^{1} \\
\text { Ricciarda Galandrini }^{2} \\
\text { Angela Santoni }{ }^{1,3} \\
\text { Rossella Paolini }^{1}{ }^{\text {iD }}
\end{array}
$$

\footnotetext{
${ }^{1}$ Department of Molecular Medicine, Laboratory affiliated to
} Istituto Pasteur Italia-Fondazione Cenci Bolognetti, "Sapienza" University of Rome, Rome, Italy ${ }^{2}$ Department of Experimental Medicine, "Sapienza" University of

Rome, Rome, Italy

${ }^{3}$ IRCCS, Neuromed, Pozzilli, Italy

Present address

Linda Quatrini, Area di Ricerca Immunologica, IRCCS Ospedale Pediatrico Bambino Gesù, Rome, Italy

\section{Correspondence}

Rossella Paolini, Department of Molecular Medicine, Laboratory affiliated to Istituto Pasteur Italia - Fondazione

Cenci Bolognetti, "Sapienza" University of Rome, "Viale Regina Elena 291, 00161, Rome, Italy. Email: rossella.paolini@uniroma1.it

\section{ORCID}

Rossella Paolini (iD https://orcid.org/0000-0001-7605-1531

Rosa Molfetta and Mario Lecce equally contributed to this work.

\section{REFERENCES}

1. Pitt JM, Kroemer G, Zitvogel L. Extracellular vesicles: masters of intercellular communication and potential clinical interventions. J Clin Invest. 2016;126:1139-1143.

2. Valadi H, Ekström K, Bossios A, Sjöstrand M, Lee JJ, Lötvall JO. Exosome-mediated transfer of mRNAs and microRNAs is a novel mechanism of genetic exchange between cells. Nat Cell Biol. 2007;9:654-659.

3. Carroll-Portillo A, Surviladze Z, Cambi A, Lidke DS, Wilson BS. Mast cell synapses and exosomes: membrane contacts for information exchange. Front Immunol. 2012;3:46.

4. Xie $\mathrm{G}$, Yang $\mathrm{H}$, Peng $\mathrm{X}$, et al. Mast cell exosomes can suppress allergic reactions by binding to IgE. J Allergy Clin Immunol. 2018;141:788-791.

5. Skokos D, Le Panse S, Villa I, et al. Mast cell-dependent B and T lymphocyte activation is mediated by the secretion of immunologically active exosomes. J Immunol. 2001;166:868-876.

6. Skokos D, Botros HG, Demeure C, et al. Mast cell-derived exosomes induce phenotypic and functional maturation of dendritic cells and elicit specific immune responses in vivo. J Immunol. 2003;170:3037-3045.

7. Mion F, D'Incà $F$, Danelli L, et al. Mast cells control the expansion and differentiation of IL-10-competent B cells. J Immunol. 2014;193:4568-4579.

8. Colombo M, Moita C, van Niel G, et al. Analysis of ESCRT functions in exosome biogenesis, composition and secretion highlights the heterogeneity of extracellular vesicles. J Cell Sci. 2013;126:5553-5565.

9. Molfetta R, Gasparrini F, Peruzzi G, et al. Lipid raft-dependent FcepsilonRI ubiquitination regulates receptor endocytosis through the action of ubiquitin binding adaptors. PLoS One. 2009;4:e5604.

\section{SUPPORTING INFORMATION}

Additional supporting information may be found online in the Supporting Information section. 